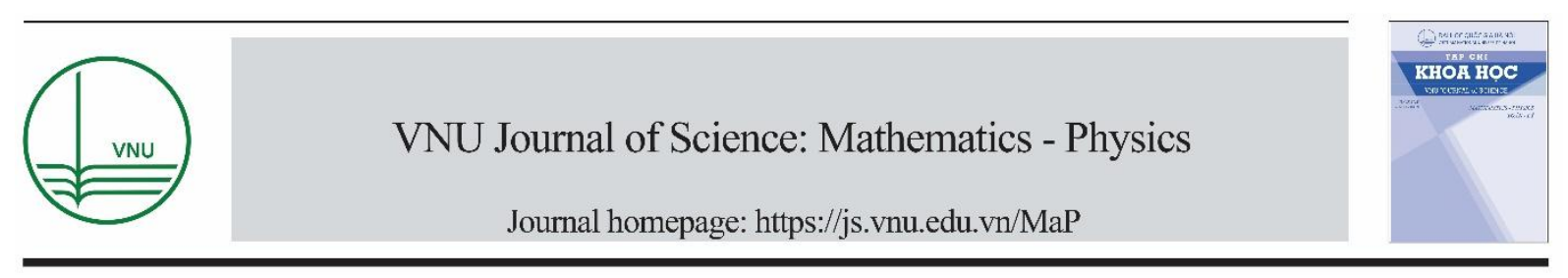

Original Article

\title{
Improvement of Critical Current Density in Bi-2223 Superconductor by Ag-Doping
}

\author{
Nguyen Khac Man, ${ }^{1, *}$, Nguyen Duc Hoa ${ }^{1}$, Duong Thi Thanh Nhan ${ }^{2}$ \\ ${ }^{1}$ International Training Institute for Materials Sience (ITIMS), \\ Hanoi University of Science and Technology, 1 Dai Co Viet, Hanoi, Vietnam \\ ${ }^{2}$ Faculty of Physics, VNU University of Science, 334 Nguyen Trai, Hanoi, Vietnam
}

Received 05 June 2019

Revised 26 June 2019; Accepted 26 June 2019

\begin{abstract}
In this study, high- $\mathrm{T}_{\mathrm{c}}$ superconducting polycrystalline $\mathrm{Bi}-2223$ undoped sample (A0) and $5 \mathrm{wt} \%$ Ag-doped sample (A5) were prepared by solid-state reaction method at $855^{\circ} \mathrm{C}$ with sintering time of 8 days. The X-ray powder diffraction (XRD) results show that the major phase of these samples was $\mathrm{Bi}-2223$. The volume ratio of $\mathrm{Bi}-2223$ phase increased from $78 \%$ for undoped sample (A0) to $95 \%$ for $5 \mathrm{wt} . \%$ Ag-doped sample (A5). The enhancement of the onset of high- $\mathrm{T}_{\mathrm{c}}$ superconductivity $\left(\mathrm{T}_{\mathrm{c} \text {,onset }}=112.5 \mathrm{~K}\right)$ in silver doping sample was observed by DC-resistivity measurements. From the AC-susceptibility measurements combined with Bean critical state model, the temperature dependent parabolic law of inter-granular or matrix critical current density $\left(\mathrm{J}_{\mathrm{c}}{ }^{\mathrm{m}}\right)$ was fitted. Some $\mathrm{J}_{\mathrm{c}}{ }^{\mathrm{m}}$ values were estimated from these parabolic laws. The results show that $5 \mathrm{wt} \% \mathrm{Ag}-$ doping can nearly double critical current density in the $\mathrm{Bi}-2223$ sample.
\end{abstract}

Keywords: Bi-2223, Ag-doping, $\mathrm{J}_{\mathrm{c}}{ }^{\mathrm{m}}$.

\section{Introduction}

Bismuth strontium calcium copper oxide, or BSCCO (pronounced "bisko"), is a family of hightemperature superconductors having the generalized chemical formula $\mathrm{Bi}_{2} \mathrm{Sr}_{2} \mathrm{Ca}_{n-1} \mathrm{Cu}_{n} \mathrm{O}_{2 n+4+\times}$. with $n=1$, 2 and 3, etc., discovered as a general class in 1988 [1], BSCCO was the first hightemperature superconductor which did not contain a rare earth element. It is a cuprate superconductor, an important category of high-temperature superconductors sharing a two-dimensional layered (perovskite)

\footnotetext{
*Corresponding author.

Email address: nkman@itims.edu.vn
}

https//doi.org/ 10.25073/2588-1124/vnumap.4357 
structure with superconductivity taking place in a copper oxide plane $\left(\mathrm{CuO}_{2}\right)$. This has been confirmed by the experiment which state that high- $\mathrm{T}_{\mathrm{c}}$ superconductivity occurs within a single $\mathrm{CuO}_{2}$ plane [2]. BSCCO and YBCO are the most studied cuprate superconductors. The superconducting properties are strongly correlating to the structural characteristics such as the increasing the superconducting transition from $36 \mathrm{~K}$ to $90 \mathrm{~K}$, and $110 \mathrm{~K}$ corresponding to number $\mathrm{n}$ of $\mathrm{CuO}_{2}$ planes Bi2101 $(\mathrm{n}=1), \mathrm{Bi} 2212(\mathrm{n}=$ $2)$ and $\mathrm{Bi} 2223(\mathrm{n}=3)$, respectively. Bilayer $\mathrm{Bi}_{2} \mathrm{Sr}_{2} \mathrm{CaCu}_{2} \mathrm{O}_{8+\mathrm{x}}(\mathrm{Bi} 2212)$ superconductor includes two homogenous $\mathrm{CuO}_{2}$ planes with $\mathrm{Cu}$ atom sitting at the center basal plane of five-oxygen pyramidal coordination. In trilayer $\mathrm{Bi}_{2} \mathrm{Sr}_{2} \mathrm{Ca}_{2} \mathrm{Cu}_{3} \mathrm{O}_{10+y}$ crystal phase ( $\left.\mathrm{Bi} 2223\right)$, there are inequivalent cuprate planes are an inner $\mathrm{CuO}_{2}$ sheet of square (four) oxygen coordination and outer $\mathrm{CuO}_{2}$ layers with a pyramidal oxygen coordination [3]. The $\mathrm{CuO}_{2}$ plane is the key parameter which determines the superconducting properties of high- $\mathrm{T}_{\mathrm{c}}$ materials such as charge density, critical transition temperature, and critical current density [4]. The properties of outer and inner layers of Bi-2223 phase are distinct. It was showed that the inner $\mathrm{CuO}_{2}$ plane has lower hole concentration and stronger antiferromagnetic spin fluctuation because of the partially screening the transferring charge from the bismuth layer (charge reservoir) to the middle $\mathrm{CuO}_{2}$ by outer $\mathrm{CuO}_{2}$ layers [5]. V. J. Emery et al. studied a system with alternating two $\mathrm{CuO}_{2}$ sheets as a model of multilayer cuprates, and suggested the strong correlation between under-doped and over-doped planes results in higher $\mathrm{T}_{\mathrm{c}}[6]$.

High-temperature superconductors are Type-II superconducting materials with rather small lower critical fields $\mathrm{H}_{\mathrm{cl}}$, usually smaller than $100 \mathrm{G}$. However, they get quite high upper critical fields $\mathrm{H}_{\mathrm{c} 2}$, about several hundreds of Tesla. On the other hand, the crystal structure with layer-by-layer structures are themselves to contain the weak links of intrinsic Josephson junctions. For polycrystalline superconductors that can be described as arrays of superconducting grains weakly coupled by Josephson junctions. Weak link behavior in the Bi-based superconducting system is the major limitation of the application at high temperatures near the transition temperature $\left(\mathrm{T}_{\mathrm{c}}\right)$. Experimentally, lower superconducting phases like $\mathrm{Bi}-2201$ and $\mathrm{Bi}-2212$ were also contributed to the grain boundaries of $\mathrm{Bi}$ 2223 particles. Therefore, to improve the quality of the superconducting material Bi-2223, the scientists have been given out several solutions. Lead was substituted for Bismuth in part to promote Bi-2223 phase content. Silver added to sample for improving the grain connections. It was found that $\mathrm{Ag}$ addition has not only affected the formation of the desired $\mathrm{Bi}-2223$ phase and the microstructure of these large bulk tube samples thereby influencing on the critical current [7]. However, Ag additions show no effects on $\mathrm{T}_{\mathrm{c}}$ and lattice parameters when samples are treated under low oxygen pressure. The formation of a low-melting eutectic liquid with $\mathrm{Ag}_{2} \mathrm{O}-\mathrm{PbO}-\mathrm{CuO}$ solid solution affects the composition of the superconducting phase and degrades superconductivity [8]. It is to be noted that $5 \mathrm{wt} . \% \mathrm{Ag}$ added Bi2223 sample showed the best microstructure with highly dense packed aligned, with quite uniform and largest grain size. As a results, it gave some better application parameters like lower critical field $\left(\mathrm{H}_{\mathrm{c} 1}\right)$, upper critical field $\left(\mathrm{H}_{\mathrm{c} 2}\right)$ as well as critical current density $\left(\mathrm{J}_{\mathrm{c}}\right)[9]$.

The critical current density is a very important technological parameter for application. This was affected by the microstructure of the superconductor. Magnetic response to AC field is more sensitive to the microstructure of the type-II superconductor than DC resistivity or static magnetization. Thus, AC susceptibility measurements have been used in both the characterization and study the vortex dynamics of high- $T_{c}$ materials. The nature of the inter-granular pinning mechanism can be investigated by AC susceptibility study since critical state models [10-12] also describe the magnetic behavior of granular high- $\mathrm{T}_{\mathrm{c}}$ superconductor. It is well known that the fundamental of the imaginary part ( $\chi$ ") of the complex AC susceptibility ( $\chi=\chi^{\prime}+\mathrm{i} \chi$ ") is a direct measure of the AC losses. In type-II superconductors, there are various loss mechanisms such as flux flow or viscous losses, bulk pinning hysteretic losses, netc. Bulk pinning losses are independent of frequency but dependent on the field, whereas flux flow losses dependent on the frequency not on the field $[11,13]$. In particularly, the imaginary component of 
the AC-susceptibility has been widely used to probe the nature of weak links in polycrystalline superconductors. It is also employed to estimate some of the important physical properties like critical current density, $\mathrm{J}_{\mathrm{c}}$ and effective volume fraction of the superconducting grains. Several critical state models have been very successful in accounting for major features of the temperature and field variation of ac-susceptibility. Experimentally, the real part of ac-susceptibility, $\chi$ ' in polycrystalline samples shows two drops as the temperature is lowered below onset of diamagnetic transition and correspondingly the derivative of the $\chi^{\prime}(\mathrm{T})$ displays two peaks. The first sharp drop at $\mathrm{T}_{\mathrm{c}}$ is due to the transition within the grains and the second gradual change at $T_{c J}$ is due to the occurrence of the superconducting coupling between grains. In the temperature range between $T_{c}$ and $T_{c J}$ the superconducting grains are decoupled and the system as a whole is resistive. Below $\mathrm{T}_{\mathrm{cJ}}$ the grains are coupled or in other terms, phase-locked with zero phase difference across the inter-granular junctions. The imaginary part, $\chi$ ”, shows a peak which is measure of the dissipation in the sample [14].

In this paper, several superconducting transition parameters, as well as inter-granular properties were investigated in two polycrystalline Bi-2223 undoped and Ag-doped samples.

\section{Experimental}

High-purity $\mathrm{Bi}_{2} \mathrm{O}_{3}, \mathrm{PbO}, \mathrm{CuO}$ oxides; and $\mathrm{SrCO}_{3}, \mathrm{CaCO}_{3}$ carbonates $(3 \mathrm{~N}-4 \mathrm{~N})$ were weighed and mixed following the nominal composition of $\mathrm{Bi}_{1.6} \mathrm{~Pb}_{0.4} \mathrm{Sr}_{2} \mathrm{Ca}_{2} \mathrm{Cu}_{3} \mathrm{O}_{10+\delta}$. The mixing powder was calcined at $800^{\circ} \mathrm{C}$ for $24 \mathrm{~h}$ with some intermediate annealing steps to improve the homogeneity. The canceled powder was divided into two equal parts (A0 and A5). The first part (A0) was compressed into rectangular pellets of $12 \mathrm{~mm}$ with quite high pressure of 5 tons per square centimeter. The second part (A5) was added with 5\% $\mathrm{Ag}_{2} \mathrm{O}$ content in weigh. After gridding, mixing we took the same steps as done with A0. After that, the pellets were sintered at $855^{\circ} \mathrm{C}$ and lasted for 8 days. XRD measurements were carried out using Bruker 5005 diffractometer with $\mathrm{Cu} \mathrm{K}_{\alpha}$ radiation $(\lambda=1.5406 \AA)$ in the range of $2 \theta=20$ $60^{\circ}$. Microstructural analyses were investigated using Jeol-5410-LV scanning electron microscope (SEM). Specimens were shaped in square bar with their dimensions of $2 \times 2 \times 12 \mathrm{~mm}^{3}$ and attached to the cold finger of a Helium closed-cycle system (CTI Cryogenic 8200) where they were cooling down and heating up in the temperature range of 20-300 K. The DC-resistivity are measured using four-probes technique with the constant DC current of $10 \mathrm{~mA}$. AC-susceptibility were performed using lock-in amplifier techniques, in different $\mathrm{AC}$ field amplitudes of 4-760 A/m at frequency of $1 \mathrm{kHz}$.

\section{Results and discussion}

\subsection{X-ray powder diffraction $(X P D)$ results}

Fig. 1. shows x-ray diffraction patterns of two superconducting samples (A0 \& A5). Almost the Bragg peaks belong to $\mathrm{Bi}-2223$ phase. In addition, the sample also consisted of some minor phases like Bi-2212 and $\mathrm{Ca}_{2} \mathrm{PbO}_{4}$. Among that $\mathrm{h}(\mathrm{ijk})$ and $\mathrm{l}(\mathrm{ijk})$ are Miller indices of the crystal planes belong to Bi2223 phase and $\mathrm{Bi}-2212$ phase, respectively. In addition, the star symbols $\left(^{*}\right)$ are also the other Bragg reflection peaks which belong to $\mathrm{Bi}-2223$ phase, too. With long sintering time of 8 days at $855^{\circ} \mathrm{C}$ it is hardly to recognize the existence of Bi-2201 phase. Besides, there are some impurity phases, especially $\mathrm{Ca}_{2} \mathrm{PbO}_{4}$ located at reflection angle $2 \theta=17.8$ degree which has seen clearly. $\mathrm{Pb}$ addition is essential for the formation of $\mathrm{Bi}-2223$ single phase but intergrowths of lower $\mathrm{T}_{\mathrm{c}}$ phases have to be avoided. Lead largely facilitates the formation of $\mathrm{Bi}-2223$ phase by reacting with $\mathrm{CaCO}_{3}$ to form a highly reactive $\mathrm{Ca}_{2} \mathrm{PbO}_{4}$ intermediate phase. Such an intermediate phase readily reacts with other constituents to form 
a liquid phase, providing a reservoir and a fast pathway for cation diffusion, and hence helping the formation of the $\mathrm{Bi}-2223$ phase [15-17]. This major impurity phase is required liquid medium for the growth of the superconducting crystallites Bi-2223 at sintering temperature. Furthermore, in A5 sample $\mathrm{Ag}$ has been found with prominent peak positioned at 38.1 degree for $\mathrm{Ag}$ (111) plane. As can be seen, both samples consist of a mixture of $\mathrm{Bi}-2223$, and $\mathrm{Bi}-2212$ phases as the major constituents. The volume fractions of these phases can be estimated using various methods. We used all the peaks of the two mentioned phases $\mathrm{Bi}-2223$ and $\mathrm{Bi}-2212$ for the characterization of the phase formation of the samples and ignore the voids [17], namely:

$$
\begin{aligned}
& B i-2223 \%=\frac{\sum I(2223)}{\sum I(2223)+\sum I(2212)} \times 100 \% \\
& B i-2212 \%=\frac{\sum I(2212)}{\sum I(2223)+\sum I(2212)} \times 100 \%
\end{aligned}
$$

Where, $\boldsymbol{I}$ is the general intensity of the present phases.

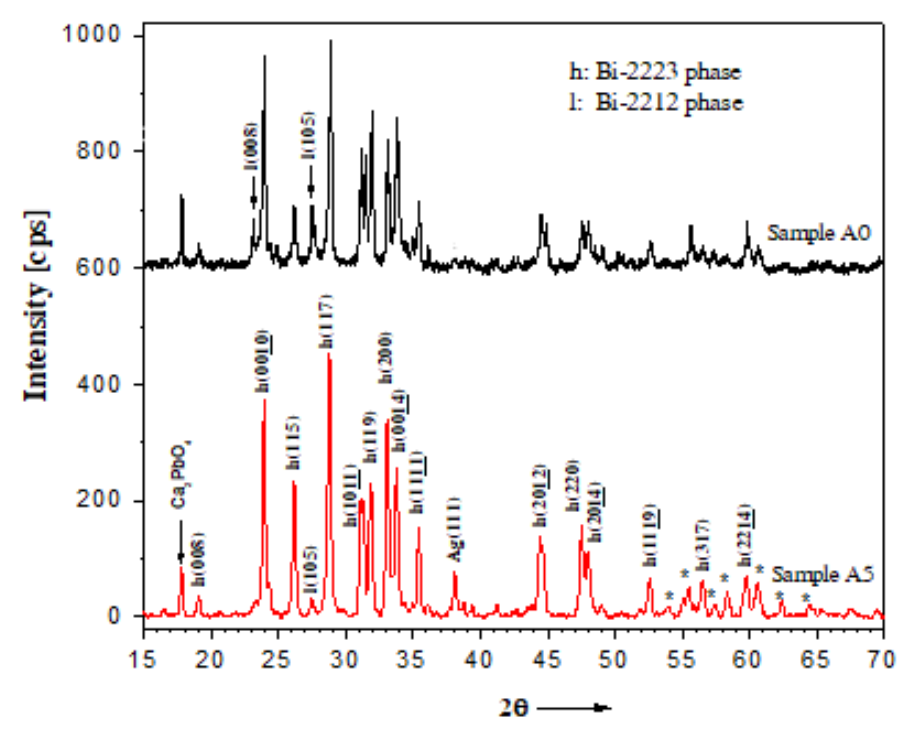

Fig. 1. XPD patterns of high- $\mathrm{T}_{\mathrm{c}}$ superconducting Bi-2223 samples: Undoped sample A0 (black line/upper graph) and 5 wt.\% Ag-doped sample A5 (red line/lower graph).

The results show that volume fraction of Bi-2223 phase increases from $78 \%$ for undoped sample (A0) to $95 \%$ for $5 \mathrm{wt} \%$ Ag-doped sample (A5). Inversely, the volume fraction of Bi-2212 phase decreases from sample A0 (22\%) to sample A5 (5\%). Therefore, Ag is positive agent to promote the growth of high- $\mathrm{T}_{\mathrm{c}}$ superconducting $\mathrm{Bi}-2223$ phase. The same evidence has been given by the author in [17]. There, highest volume fraction of Bi-2223 phase existed in the Ag interface layer. The crystal structure of Bi-2223 phase is pseudo-tetragonal unit cell (I4/mmm). The crystal lattices of undoped sample $\mathrm{A} 0$ are $\mathrm{c}=37.109 \AA$ and $\mathrm{a} \sim \mathrm{b}=5.402 \AA$. These parameters are unchanged in caparison to those of Ag-doped sample (A5). When chose Ag substitution for Sr, Ghazala et al conclude that Ag dose not deteriorate the superconducting properties of the BSCCO system but plays a crucial role in the stabilization of the different phases coexisting in the Bi-based superconductors. This result indicates 
that the Ag has catalytic effect on the reaction to form the high-Tc phase within the $\mathrm{x}$ value equal to 0.05 and 0.1 . However, certain amount of $\mathrm{Ag}$ is necessary for the occurrence of this reaction, while excessive Ag substitution promotes another reaction, assist the formation of low $\mathrm{T}_{\mathrm{c}}$ phase [18].

\subsection{Scanning electron microscope (SEM) images}

Both samples obtained after a quite long time of sintering ( 8 days) have strong dark-gray color with many micro-size crystals, which can be seen by the eyes. With Bi-2223 is major phase, we suggest that the micro-size crystalline particles to be Bi-2223 crystals, especially for A5 sample. The size of these crystalline plates (grains) is in the range of $5-10 \mu \mathrm{m}$. These thin plates with $\mathrm{CuO}_{2}$-plane preferential growth pack together to form the samples (see Fig.2). It was found that the Bi-2212 phase on the grain boundaries is likely to play the role of weak-link and consequently reduces the inter-granular critical current densities [8]. However, Ag plays the role to promote the growth rate of Bi-2223 phase, as well as to enhance the connectivity of these grains were obtained, which was evidenced by less porosities in A5 sample. The scanning electron micrographs indicated that silver is precipitated along the grain boundaries, separating the superconducting grains [19]. These results have showed that silver addition does not destroy the superconductivity and at the same time enhances the critical current density.

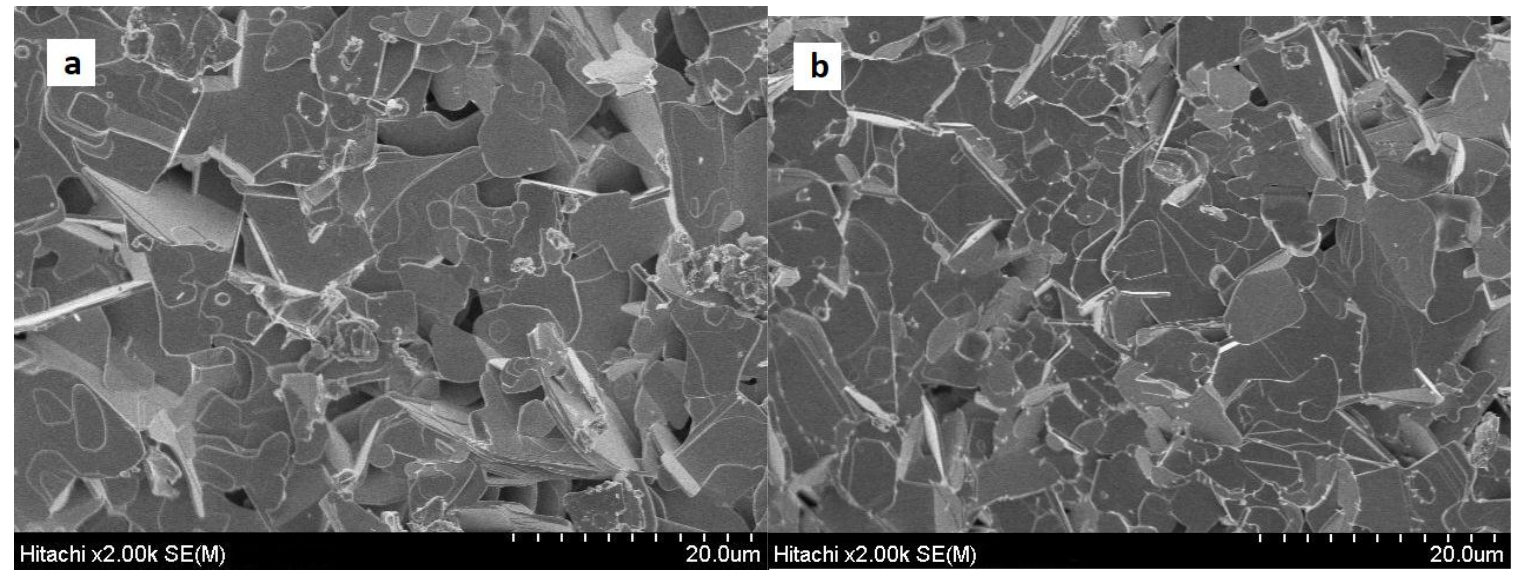

Fig. 2. SEM pictures of the Bi2223 superconductors: Sample A0 (a-left) and sample A5 (b-right).

\subsection{DC-resistivity results}

The relative resistivity $\mathrm{R}(\mathrm{T}) / \mathrm{R}(300 \mathrm{~K})$ of the $\mathrm{Bi}-2223$ samples were depicted in Fig.3. Both samples show the metallic behavior of the resistance at high temperature region. As decreasing temperature, a transition to the superconducting state occurred. These curves describe the typical resistivity of Bi-2223 superconducting sample. However, when $\mathrm{Ag}$ was doped the resistivity strongly dropped. Some resistivity values at 120 and $300 \mathrm{~K}$ were present in Table 1 . As can be seen in Fig. 3, the metallic characteristics of sample A5 was improved by Ag-doping. These were explained by the diffusion of silver in the samples. A detailed investigation about diffusion of Ag in BSCCO system has been done by Comert et al [20] who suggested that Ag can diffuse simultaneously into the voids, cracks, intergrain/intragrain regions etc. This improves the grain orientation and intergrain connectivity in the samples. Study of the superconducting transition region should be very important. There are different ways to define the transition temperature $\left(T_{c}\right)$. Therefore, we have chosen to study the superconducting transition range by taking the differential of the resistivity curves (see the inset figure included in Fig. 
3). The derivative curves show that there are three main particular points $T_{c}$,onset, $T_{c}$ and $T_{c, 0}$ corresponding. Where $T_{c, 0}$ is the temperature of zero-resistivity; $T_{c}$ is temperature of differential peak. We assign $T_{c}$,onset for the starting point of superconducting coherence or superconducting transition. In undoped sample, there is only a single peak. However, there appears a peak with a tail $(*)$ in Ag-doped sample. The peak temperature marks the superconducting transition within the grains and the tail is related to the inter-granular coupling [21]. The transition parameters were given in Table 1. The onset of high-Tc superconductivity ( $\mathrm{T}_{\mathrm{c}, \text { onset }}$ ) was enhanced $3 \mathrm{~K}$ by silver doping from 109.5 up to $112.5 \mathrm{~K}$. The superconducting transition temperature was defined as the differential peak position of resistivity $\left(\mathrm{T}_{\mathrm{c}}\right)$. This value was nearly unchanged by $5 \%$ at silver doping $(\sim 105 \mathrm{~K})$. However, the zero-resistance temperature $\left(\mathrm{T}_{\mathrm{c}, 0}\right)$ was declined to $98.4 \mathrm{~K}$ for A5 sample. As a results, the superconducting transition region of 5\% at. Ag-doped sample has been extended with $\sim 5 \mathrm{~K}$ in comparison with that of undoped sample. The increase of the onset point of temperature may be explained by the strong high- $\mathrm{T}_{\mathrm{c}}$ superconductivity of Bi-2223 phase accompanied by the decrease of Bi-2212 volume fraction.

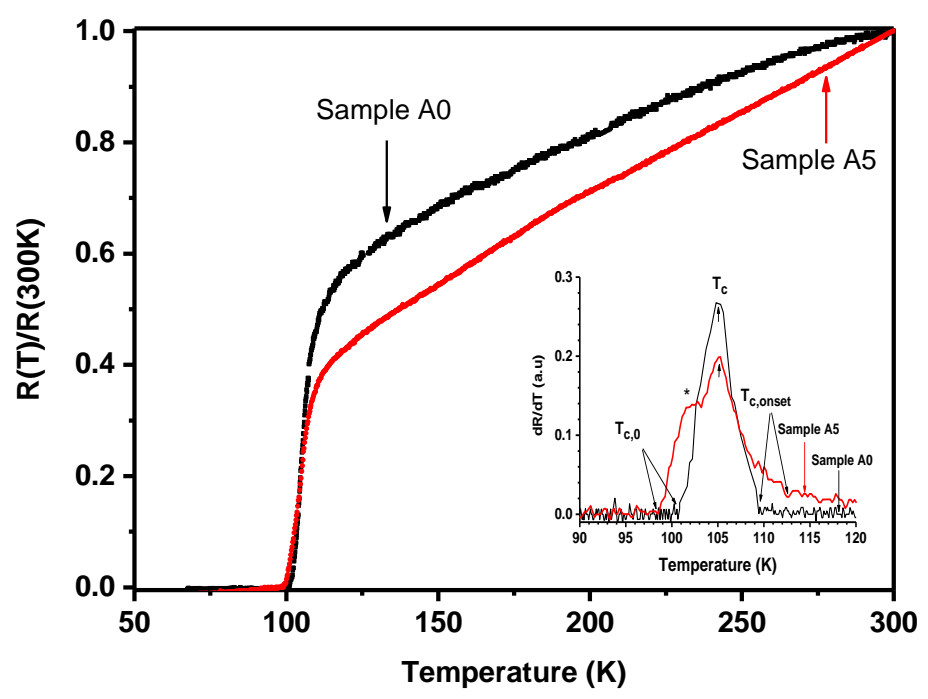

Fig. 3. Describe relative resistivity $\mathrm{R}(\mathrm{T}) / \mathrm{R}(300 \mathrm{~K})$ versus temperature of the Bi-2223 Samples: The black line (sample A0) and the red line (sample A5). Inset shows the temperature dependence of first derivatives taken from the relative resistivity curves, respectively.

The substitution of $\mathrm{Ag}+(\mathrm{r}=1.29 \AA \hat{)})$ for constituent cations may be suitable for $\mathrm{Sr}^{2+}(\mathrm{r}=1.32 \AA \AA)$. This substitution should be taken place by the slowly diffusion from the boundary of high-Tc grains because of the chemical mismatch. As usually, the air-grown Bi-2223 samples have the hole concentration in under-doped region. The silver substitution for strontium may be created the positive effect on the hole content of $\mathrm{CuO}_{2}$ planes. As a results, the onset of high-Tc superconducting coherence arrived soon or $\mathrm{T}_{\mathrm{c}, \text { onset }}$ become higher in Ag-doped sample. Although silver make the positive effect on $\mathrm{T}_{\mathrm{c}, \text { onset, }}$ It also make the negative effect on $\mathrm{T}_{\mathrm{c}, 0}$. The lower zero-resistance transition temperature may be explained by the presence of silver in the superconducting grain boundaries. With different sizes and shapes, the high-Tc superconducting grains connect each other by the grain boundary materials including silver. It is the reason for creating the proximity coupling effects of the superconducting grains. As consequence, the total superconducting effect of silver-doped sample was taken place at lower temperature. The same effect was observed in Li-doped Bi-2223 system [22]. 
Table 1. Superconducting transition parameters and resistivity values of Bi-2223 samples A0\&A5 taken from DC-resistivity curves

\begin{tabular}{llllcll}
\hline Sample & \multicolumn{3}{c}{ Transition } & parameters & Resistivity & $(\mathrm{m} \Omega . \mathrm{cm})$ \\
\hline & $\mathrm{T}_{\mathrm{c}, 0}(\mathrm{~K})$ & $\mathrm{T}_{\mathrm{c}}(\mathrm{K})$ & $\mathrm{T}_{\mathrm{c}, \text { onset }}(\mathrm{K})$ & $\Delta \mathrm{T}_{\mathrm{c}}(\mathrm{K})$ & $\rho(300 \mathrm{~K})$ & $\rho(120 \mathrm{~K})$ \\
A0 & 100.3 & 105.0 & 109.5 & 9.2 & 8.52 & 4.85 \\
A5 & 98.4 & 105.1 & 112.5 & 14.1 & 3.28 & 1.41 \\
\hline
\end{tabular}

\subsection{AC-susceptibility and matrix critical current density $\left(J_{c}{ }^{m}\right)$ values}

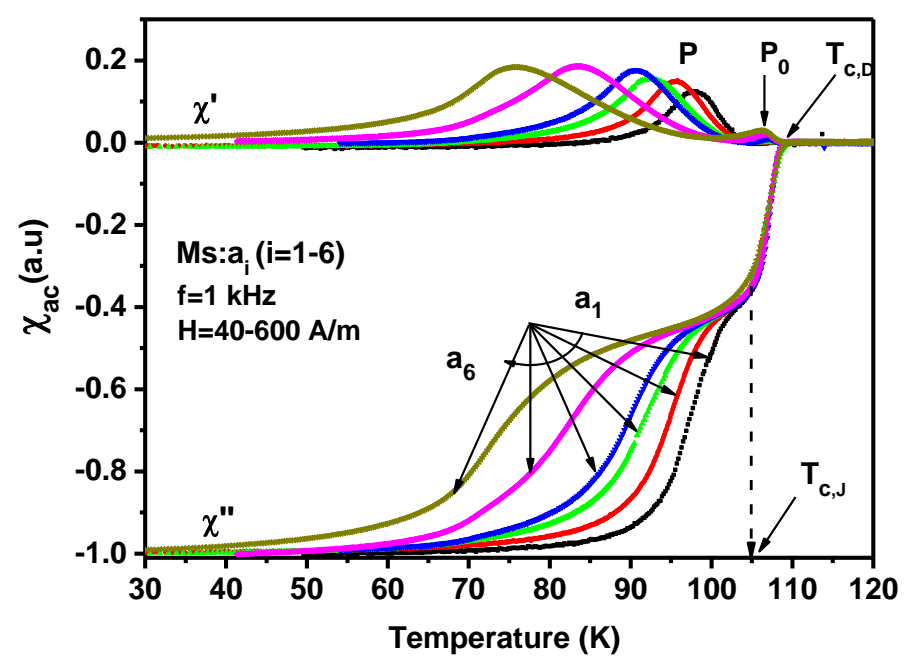

Fig. 4. Temperature dependence of the ac-susceptibility of the superconducting Bi-2223 sample A0 in various AC field amplitudes at a frequency of $1 \mathrm{kHz}$. The ac-susceptibility measurements ai (i=1-6) are corresponding to AC field amplitudes (40, 80, 132, 180, 360, and $600 \mathrm{~A} / \mathrm{m})$, respectively.

The magnetic curves in Figures $4 \& 5$ were assigned to the ac-susceptibilities of the high- $\mathrm{T}_{\mathrm{c}} \mathrm{Bi}-2223$ samples A0 \& A5 in various AC field amplitudes $(4-760 \mathrm{~A} / \mathrm{m})$ at a frequency of $1 \mathrm{kHz}$. The AC fields in this work are all given as rms. values. For undoped sample A0, the temperature at which the diamagnetic signal $\left(\chi^{\prime}\right)$ get dropping is approximately $109.5 \mathrm{~K}\left(\mathrm{~T}_{\mathrm{c}, \mathrm{D}}\right)$. $\mathrm{T}_{\mathrm{c}, \mathrm{D}}$ is also the starting point of of upturn curve in imaginary part ( $\chi$ ”) when AC field is high enough to penetrate into the superconducting grains. This temperature value is equal to that of the onset of high-Tc superconductivity $\left(T_{c, \text { onset }}\right)$ in resistivity measurement. However, $\mathrm{T}_{\mathrm{c}, \mathrm{D}}$ was decreased to lower temperature of $108.6 \mathrm{~K}$ in Ag-doped sample (A5). In addition, the diamagnetic onset temperature is approximately constant at different fields for both samples.

The real part ( $\left.\chi^{\prime}\right)$ curves of AC-susceptibility all have been divided by the cross point $\mathrm{M}$ into two parts. $\mathrm{T}_{\mathrm{M}}$ is approximately equal to $105 \mathrm{~K}$ for both samples. The first one in the temperature range of $\left(T_{c, D}-T_{c, J}\right)$ can be attributed to the intra-grain diamagnetic signal, and the second one at lower temperature ascribed to inter-grain part. In the temperature range between $T_{c, D}$ to $T_{c, J}$ the superconducting grains are decoupled and the system as a whole is resistive. Below $T_{c, J}$ the grains are coupled or in other terms, phase-locked with zero phase difference across the intergranular junctions 
[14]. The more the AC-magnetic amplitude apply, the more deflection of intra-grain part to lower diamagnetic signal is. The deflection is accompanied by appearance of loss peak $\mathrm{P}_{0}$ of intra-grain component. It is clearly from Fig. 4 \&5 that when the field increases, the loss peak of imaginary part (P) shifts to lower temperature and broadens.

Table 2. The peak temperatures corresponding to the field amplitudes $\mathrm{H}_{\mathrm{ac}}$ and inter-granular critical current density $\mathbf{J}_{\mathrm{c}}{ }^{\mathrm{m}}$ in different measurements $\mathrm{a}_{\mathbf{i}}(\mathbf{i}=\mathbf{1 - 6})$ of undoped sample A0

\begin{tabular}{lllll}
\hline $\mathrm{j}=$ & $\mathrm{H}_{\mathrm{ac}}(\mathrm{A} / \mathrm{m})$ & $\mathrm{T}_{\mathrm{p} 0}(\mathrm{~K})$ & $\mathrm{T}_{\mathrm{p}}(\mathrm{K})$ & $\mathrm{J}_{\mathrm{c}}{ }^{\mathrm{m}}\left(\mathrm{A} / \mathrm{cm}^{2}\right)$ \\
\hline 1 & 40 & 107.3 & 98.1 & 2 \\
2 & 80 & 107.3 & 95.7 & 4 \\
3 & 132 & 107.3 & 92.6 & 6.5 \\
4 & 180 & 107.2 & 90.8 & 9 \\
5 & 360 & 106.6 & 84.1 & 18 \\
6 & 600 & 106.0 & 76.0 & 30 \\
\hline
\end{tabular}

The amount of shift as a function of the magnetic field amplitude $\left(\mathrm{H}_{\mathrm{ac}}\right)$, is proportional to the magnitude or strength of the pinning force. At any certain AC-magnetic field, when the temperature was decreased, the flux is gradually driven out of the superconducting grains, then the inter-granular volume. The full Meissner effect happened when the applied magnetic field totally excluded. At this temperature, the whole volume of the sample expected to be shielded by the super-current circulating in the sample and hence the diamagnetic signal becomes saturation. In case for the lowest AC-field amplitude $(\mathrm{H}=4$ $\mathrm{A} / \mathrm{m})$ in Fig.5 the diamagnetic signal attained at $85 \mathrm{~K}$. This result gives evidence of the minor un-definable content of Bi-2212 phase. When magnetic field increases, the ideal diamagnetic signals appear at lower temperatures. As can be seen in Table $2 \& 3$, the positions of intra-grain peak $\left(\mathrm{T}_{\mathrm{p} 0} \sim 107.2\right.$ - $107.3 \mathrm{~K})$ are nearly constant at low AC-field values $(<180 \mathrm{~A} / \mathrm{m})$, accompanied by the small areas of intra-grain loss peaks. In contrary, this effect become clearly at higher magnetic fields $(360-760 \mathrm{~A} / \mathrm{m})$, as well as the shift of $\mathrm{T}_{\mathrm{p} 0}$ down to $106.0 \mathrm{~K}$. Nevertheless, this intra-grain loss effect is quite smaller than that observed by inter-grain effect.

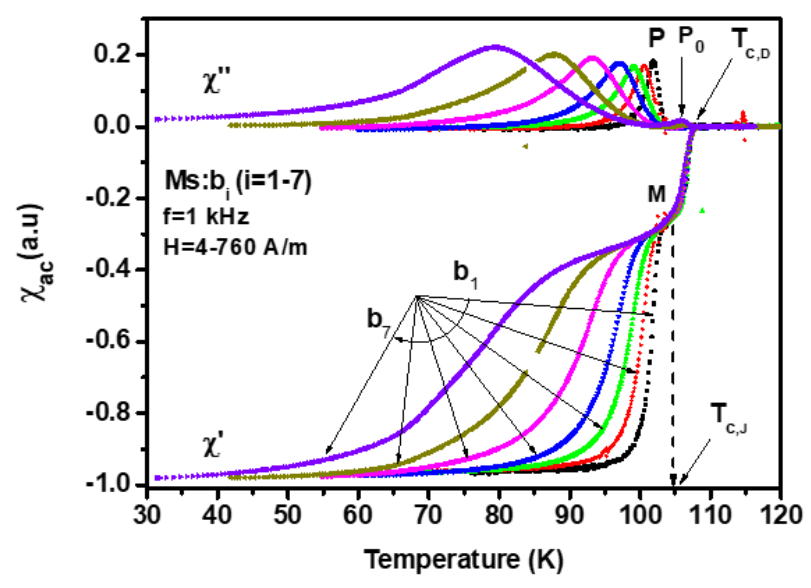

Fig. 5. Temperature dependence of the ac-susceptibility of the superconducting Bi-2223 sample A5 in various AC field amplitudes at a frequency of $1 \mathrm{kHz}$. The ac-susceptibility measurements $b_{i}(i=1-7)$ are corresponding to AC field amplitudes $(4,12,40,80,200,360$, and $760 \mathrm{~A} / \mathrm{m})$, respectively. 
Table 3. The peak temperatures corresponding to the field amplitudes $\mathrm{H}_{\mathrm{ac}}$ and inter-granular critical current density $\mathbf{J}_{\mathfrak{c}}{ }^{\mathrm{m}}$ in different measurements $\mathbf{b}_{\mathbf{i}}(\mathbf{i}=\mathbf{1 - 7})$ of Ag-doped sample A5

\begin{tabular}{lclll}
\hline $\mathrm{i}=$ & $\mathrm{H}_{\mathrm{ac}}(\mathrm{A} / \mathrm{m})$ & $\mathrm{T}_{\mathrm{p} 0}(\mathrm{~K})$ & $\mathrm{T}_{\mathrm{p}}(\mathrm{K})$ & $\mathrm{J}_{\mathrm{c}} \mathrm{m}\left(\mathrm{A} / \mathrm{cm}^{2}\right)$ \\
\hline 1 & 4 & 107.2 & $101.9 \mathrm{~K}$ & 0.2 \\
2 & 12 & 107.2 & $100.6 \mathrm{~K}$ & 0.6 \\
3 & 40 & 107.2 & $99.4 \mathrm{~K}$ & 2 \\
4 & 80 & 107.2 & $97.2 \mathrm{~K}$ & 4 \\
5 & 200 & 106.7 & $93.8 \mathrm{~K}$ & 10 \\
6 & 360 & 106.0 & $88.0 \mathrm{~K}$ & 18 \\
7 & 760 & 106.0 & $80.0 \mathrm{~K}$ & 38 \\
\hline
\end{tabular}

In the polycrystalline samples like Bi-2223, the weak-link is one of dominant property that affect strongly on the superconducting properties as well as the working point, especially the critical current density. When increase the AC-magnetic amplitude, the loss peak $(\mathrm{P})$ shifts to lower temperature and broadens. We can estimate the inter-granular or matrix critical current density $\left(\mathrm{J}_{\mathrm{c}}{ }^{\mathrm{m}}\right)$ as a function of temperature, by using the Bean critical state model [12]. According to this model for the bar shaped sample, $\mathrm{J}_{\mathrm{c}}{ }^{\mathrm{m}}$ at the peak temperature $\left(\mathrm{T}_{\mathrm{p}}\right)$, can be written as $\mathrm{J}_{\mathrm{c}}{ }^{\mathrm{m}}=\mathrm{H}_{\mathrm{ac}}(\mathrm{WxD})^{1 / 2}$, where the cross section of the rectangular bar shaped sample is $2 \mathrm{Wx} 2 \mathrm{D}$. The larger the shift in the maxima of $\chi$ ", the weaker the pinning and hence, the smaller $\mathrm{J}_{\mathrm{c}}{ }^{\mathrm{m}}$. Data of $\mathrm{J}_{\mathrm{c}}{ }^{\mathrm{m}}$ were given from Table $2 \& 3$ and presented in Fig. 6 . The stronger curvature of $\mathrm{J}_{\mathrm{c}}{ }^{\mathrm{m}}$ plot of silver-doped sample A5 in comparison to that of un-doped sample A0 gave us the faster increase of inter-granular or matrix critical current density when the temperature was decreased. Therefore, silver doping at $5 \mathrm{wt}$ \% in Bi-2223 make the superconducting sample much better for application. We can calculate the $\mathrm{J}_{\mathrm{c}}{ }^{\mathrm{m}}$ at boiling point of liquid nitrogen $(77 \mathrm{~K})$ to be 28.5 and 47 $\mathrm{A} / \mathrm{cm}^{2}$ for $\mathrm{A} 0$ and $\mathrm{A} 5$ samples, respectively. This critical current density can be estimated to go up to 88 and $162 \mathrm{~A} / \mathrm{cm}^{2}$ at $50 \mathrm{~K}$. These results are in comparison to $\mathrm{J}_{\mathrm{c}}{ }^{\mathrm{m}}$ values of the best sample in ref. [14]. However, there are some other factors which may effect to this key application parameter at lower temperature. Normally, for Bi-based high- $\mathrm{T}_{\mathrm{c}}$ superconductors the critical current density increase following the exponential law.

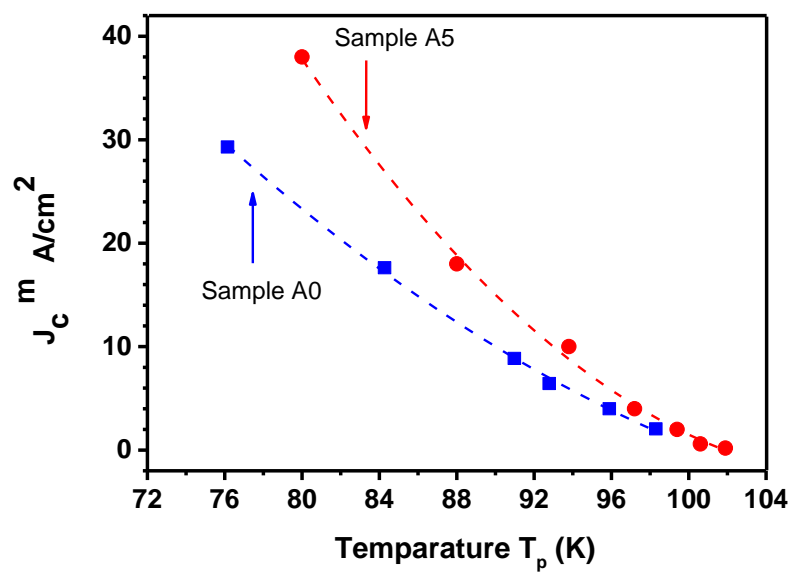

Fig. 6. Shows the inter-granular or matrix critical current density $\left(\mathrm{J}_{\mathrm{c}}{ }^{\mathrm{m}}\right)$ as a function of temperature for sample A0 (blue rectangular) and sample A5 (red circle). Both curves were fitted well to the parabolic law of $\mathrm{T}_{\mathrm{p}}$ with the formulas: $\mathrm{J}_{\mathrm{c}}{ }^{\mathrm{m}}=273.15-4.68 \mathrm{~T}_{\mathrm{p}}+0.0195 \mathrm{~T}_{\mathrm{p}}{ }^{2}$ for $\mathrm{A}_{0}$ and $\mathrm{J}_{\mathrm{c}}{ }^{\mathrm{m}}=553.20-10.14 \mathrm{~T}_{\mathrm{p}}+0.0463 \mathrm{~T}_{\mathrm{p}}{ }^{2}$ for A5 samples, respectively. 


\section{Conclusion}

At the same sintering condition, the growth rate of $\mathrm{Bi}-2223$ phase was investigated to increase by $\mathrm{Ag}$-doping. Specifically, the volume ratio of $\mathrm{Bi}-2223$ phase increase from $78 \%$ for undoped sample (A0) to $95 \%$ for 5 wt.\% Ag-doped sample (A5). Herein, Ag plays the role to promote the growth rate of Bi2223 phase, as well as to enhance the connectivity of these grains were obtained, which was evidenced by less porosities in A5 sample. As consequence, the inter-granular or matrix critical current density $\left(\mathrm{J}_{\mathrm{c}}{ }^{\mathrm{m}}\right)$ was observed to be increased. We can calculate the $\mathrm{J}_{\mathrm{c}}{ }^{\mathrm{m}}$ at boiling point of liquid nitrogen $(77 \mathrm{~K})$ to be 28.5 and $47 \mathrm{~A} / \mathrm{cm}^{2}$ for A0 and A5 samples, respectively. This critical current density can be estimated to go up to 88 and $162 \mathrm{~A} / \mathrm{cm}^{2}$ at $50 \mathrm{~K}$. The onset of high- $\mathrm{T}_{\mathrm{c}}$ superconductivity $\left(\mathrm{T}_{\mathrm{c}, \text { onset }}\right.$ ) was enhanced 3 $\mathrm{K}$ by silver doping from 109.5 up to $112.5 \mathrm{~K}$. It is explained by the slowly substitution of $\mathrm{Ag}^{+}$ions for $\mathrm{Sr}^{2+}$ cations. As a results, it increases the hole concentration of $\mathrm{CuO}_{2}$ planes of Bi-2223 phase, and hence the onset of high- $T_{c}$ superconductivity. Nevertheless, the metallic silver at the grain boundaries also cause the proximity effect to weaken the superconductivity of the sample $\left(\mathrm{T}_{\mathrm{c}, 0}\right)$.

\section{Acknowledgements}

The current work was financially supported by the HUST Science \& Technology Project (20172018, Code: T2017-PC-174).

\section{References}

[1] H. Maeda, Y. Tanaka, M. Fukutumi, T. Asano, A new high- $\mathrm{T}_{\mathrm{c}}$ oxide superconductor without a rare earth element, Jpn. J. Appl. Phys. 27 (2) (1988) L209.

[2] G. Logvenov, A. Gozar, I. Bozovic, High-Temperature Superconductivity in a Single Copper-Oxygen Plane, Science 326, (2009) 699-702 https://doi.org/10.1126/science.1178863.

[3] Takenori Fujii, Ichiro Terasaki, Takao Watanabe, Azusa Matsuda, Doping dependence of anisotropic resistivities in the trilayered superconductor $\mathrm{Bi}_{2} \mathrm{Sr}_{2} \mathrm{Ca}_{2} \mathrm{Cu}_{3} \mathrm{O}_{10+\delta}$, Phys. Rev. B 66 (2002) 024507, https://doi.org/10.1103/PhysRevB.66.024507.

[4] E. Pavarini, T.S. Dasgupta, O. Jepsen, O.K. Adersen, Band-Structure Trend in Hole-Doped Cuprates and Correlation with $\mathrm{T}_{\text {cmax }}$, Phys. Rev. Lett. 87 (2001) 047003, https://doi.org/10.1103/PhysRevLett.87.047003

[5] B.W. Statt, L.M. Song, Screening of the middle $\mathrm{CuO}_{2}$ layer in $\mathrm{Bi}_{1.6} \mathrm{~Pb}_{0.4} \mathrm{Sr}_{2} \mathrm{Ca}_{2} \mathrm{Cu}_{3} \mathrm{O}_{10}$ determined from $\mathrm{Cu} \mathrm{NMR}$, Phys. Rev. B 48 (1993) 3536-3539, https://doi.org/10.1103/PhysRevB.48.3536.

[6] V.J. Emery, S.A. Kivelson, Importance of phase fluctuations in superconductors with small superfluid density, Nature 374 (1995) 434-437, doi:10.1038/374434a0.

[7] S.N. Ekbote, G.K. Padam, M. Sharma, N.K. Arora, B.S. Khurana, R.C. Goel, D.K. Suri, N. Mehra, B.K. Das, Preparation and characterization of Ag-added $\mathrm{Bi}_{1.84} \mathrm{~Pb}_{0.4} \mathrm{Sr}_{2} \mathrm{Ca}_{2.2} \mathrm{Cu}_{3} \mathrm{O}_{10+\mathrm{x}}$ bulk tube conductors for cryogen free superconducting magnet, Bull. Mater. Sci., Vol. 24, No. 6, (2001) 603-609, https://doi.org/10.1007/BF02704008

[8] S.X. Dou, K.H. Song, H.K. Liu, C.C. Sorrell, M.H. Apperly, N. Savvides, Superconductivity in a Ag- doped BiPb- Sr- Ca- Cu- O system, Appl. Phys. Lett. 56, (1990) 493-494, https://doi.org/10.1063/1.103298.

[9] T.D. Hien, N.D. Minh, N.K. Man, N.H. Sinh, Some critical characteristics of Ag-doped Bi-2223 superconductors from magnetic hysteresis loop measurements, Physics \& Engineering in Evolution, Proceedings of the Fifth Vietnamese-Greman Seminar on Physics and Engineering, No. 188/2002/XBNT/VHTT, Hanoi (2002) 136-140.

[10] S.A. Halim, S.A. Khawaldeh, S.B. Mohamed, A. Azhan, Superconducting properties of $\mathrm{Bi}_{2-x} \mathrm{~Pb}_{\mathrm{x}} \mathrm{Sr}_{2} \mathrm{Ca}_{2} \mathrm{Cu}_{3} \mathrm{O}_{\mathrm{y}}$ system derived via sol-gel and solid state routes, Mater. Chem. \& Phys. 61, (1999) 251-259.

[11] C.Y. Lee, Y.H. Kao, Frequency dependence of the intergranular energy-loss peak in AC magnetic susceptibility of high- $T_{\mathrm{c}}$ superconductors, Physica C 256, (1996) 183-190, https://doi.org/10.1016/0921-4534(95)00639-7. 
[12] C.B. Bean, Magnetization of High-Field Superconductors, Rev. Mod. Phys. 36 (1964) 31-39, https://doi.org/10.1103/RevModPhys.36.31.

[13] S. Celebi, I. Karaca, E. Aksu, A. Grencer, Frequency dependence of the intergranular AC loss peak in a high- $T_{\mathrm{c}} \mathrm{Bi}-$ $(\mathrm{Pb})-\mathrm{Sr}-\mathrm{Ca}-\mathrm{Cu}-\mathrm{O}$ bulk superconductor, Physica C 309, (1998) 131-137, https://doi.org/10.1016/S09214534(98)00531-0.

[14] H. Salamati, P. Kameli, The effect of Bi2212 phase on the weak link behavior of Bi2223 superconductors, Physica C 403 (2004) 60-66, DOI: 10.1016/j.physc.2003.11.009.

[15] M.H. Mohammed, R. Awad, A.I. Abou-Aly, I.H. Ibrahim, M.S. Hassan, Optimizing the Preparation Conditions of Bi-2223 Superconducting Phase Using $\mathrm{PbO}$ and $\mathrm{PbO}_{2}$, Materials Sciences and Applications 3 (2012) 224-233, DOI: $10.4236 / \mathrm{msa} .2012 .34033$.

[16] O. Bilgili, Y. Selamet, K. Kocabas, Effects of Li Substitution in Bi-2223 Superconductors, J. Supercond. Nov. Magn. 21 (2008) 439-449, DOI: 10.1007/s10948-008-0374-4.

[17] A.V. Narlikar, Studies of high temperature superconductors, Vol. 35 (The BSCCO system), Nova Science Publishers, Inc, ISNB 1-5672-789-6, New York, (2000).

[18] A. Oota, A. Kirihigashi,Y. Sasaki, K. Ohba, Growth Process of the (2223) Phase in Pb-Added Bi-Sr-Ca-Cu-O, Japn. J.Appl. Phys. 27 (1988) L2289, https://doi.org/10.1143/JJAP.28.L1171.

[19] M. Muralidhar, K.N. Kishore, M. Nagashushanam, V.H. Babu, Role of Silver on Superconducting Properties of BPSCCO (2223) System, Crys. Res. Technol. 28, (1993) 653-663, https://doi.org/10.1002/crat.2170280515.

[20] H. Comert, M. Altunbag, T.D. Dzhafarov, T. Kusukomeroglu, Y.G. Asadov, H. Karal, The effect of Ag diffusion on the crystal structure and electrical properties of $\mathrm{Bi}(\mathrm{Pb}) \mathrm{SrCaCuO}$ superconductors, Supercond. Sci. Technol.7 (1994) 824-827, https://doi.org/10.1088/0953-2048/7/11/009.

[21] P. Kameli, H. Salamati, M. Eslami, The effect of sintering temperature on the intergranular properties of Bi2223 superconductors, Solid State Commun. 137 (2006) 30-35, https://doi.org/10.1016/j.ssc.2005.10.026.

[22] Nguyen Khac Man, Enhancement of Superconducting Critical Temperature in $\mathrm{Bi}(\mathrm{Pb})-\mathrm{Sr}-\mathrm{Ca}-\mathrm{Cu}-\mathrm{O}$ System by Li-Doping, Journal of Science \&Technology 135 (Technical Universities) (2019) 60-66. 